\title{
Discrepâncias na imagem corporal e na dieta de obesos
}

\section{Self-discrepancy in body image and diet}

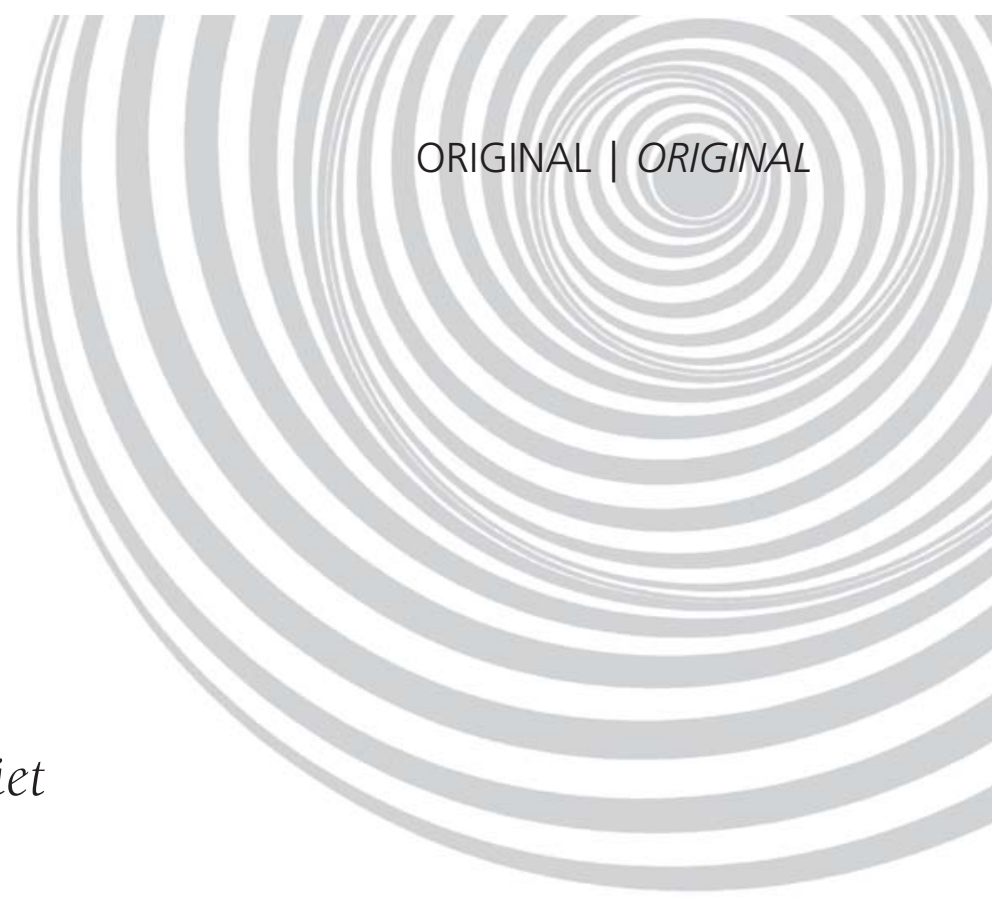

Patrícia KANNO ${ }^{1}$

Misael RABELO'

Gislane Ferreira de MELO'

Adriana GIAVONI ${ }^{1}$

\section{RE S U M O}

\section{Objetivo}

Este estudo avaliou a discrepância entre a imagem real e a imagem ideal de indivíduos obesos e procurou relacionar possíveis alterações no comportamento alimentar na busca desse corpo ideal.

\section{Métodos}

A amostra foi composta por 25 sujeitos, sendo 76\% do sexo feminino e idade média de 39,24 (desviopadrão=5,01) anos. Dois instrumentos foram utilizados: a Escala de Aparência Física, cuja análise fatorial extraiu um único fator "Aparência Física" com precisão de $\alpha=0,74$ para mulheres e $\alpha=0,73$ para homens e o Questionário de Prioridade Alimentar elaborado para agrupar itens nas categorias que compõem a Pirâmide Alimentar. Foram realizados testes $t$ pareados para comparar diferenças entre as imagens real e ideal e para comparar os comportamentos alimentares real e ideal.

\section{Resultados}

Os resultados apresentaram diferenças entre as imagens ideal e real, sendo a primeira representada de forma mais positiva que a última. Com relação ao comportamento alimentar, os resultados demonstraram diminuição no consumo de carnes e café preto e aumento no consumo de frutas e vegetais para atingir o corpo ideal. Porém, a amostra não mudaria os seus hábitos em relação ao consumo de cereais, laticínios, óleos e gorduras, doces e refrigerantes.

\section{Conclusão}

Embora os resultados apresentem diferenças na percepção da imagem corporal, a amostra não mudaria os seus hábitos em relação às categorias do topo da pirâmide alimentar.

Termos de indexação: Comportamento alimentar. Educação alimentar e nutricional. Obesidade. Nutrição de grupos de risco.

\footnotetext{
1 Universidade Católica de Brasília, Pós-Graduação Stricto Sensu em Educação Física. QS 07, lote 01, EPCT, 71966-700, Águas Claras, Brasília, DF, Brasil. Correspondência para/Correspondence to: A. GIAVONI. E-mail: <adriana@pos.ucb.br>.
} 
424 P. KANNO et al.

\section{A B S T R A C T}

\section{Objective}

This study evaluated the discrepancy between actual and ideal body images of obese individuals and listed changes in the dietary behaviors used to achieve this ideal body image.

\section{Methods}

The sample was composed of 25 obese individuals with a mean age of 39.24 years (standard deviation=5.01) where $76 \%$ were females. Two instruments were used for this end: the Physical Appearance Scale whose factorial analysis extracted a single factor "Physical Appearance" with an accuracy of $\alpha=0.74$ for women and $\alpha=0.73$ for men and the Food Priority Questionnaire which was developed to group items in categories of the food pyramid. Paired T-tests were done to compare differences between actual and ideal body images and actual and ideal eating behaviors.

\section{Results}

The results revealed differences between ideal and actual body images and the former was more positive than the latter. Regarding dietary behavior, the results showed that consumption of meats and black coffee diminished and consumption of fruits and vegetables increased to achieve the ideal body image. However, the sample would not change their dietary habits regarding the consumption of grains, dairy, oils and fats, sweets and soft drinks.

\section{Conclusion}

Although, the results showed differences in perception of body image, the sample would not change their habits regarding the categories on the top of the food pyramid.

Indexing terms: Feeding behavior. Food and Nutrition Education. Obesity. Risk Groups Nutrition.

\section{N T R O D U ÇÃ O}

Para a Psicologia Social, o autoconceito é a autoconstrução que o indivíduo elabora a respeito de si ao longo da vida. É uma estrutura central, composta por cognições, afetos, sentimentos, comportamentos e atitudes que o indivíduo possui a respeito de si mesmo, dos outros e do meio em que vive ${ }^{1,2}$. Esta estrutura é formada a partir das percepções, conjecturas e imaginações que o indivíduo realiza a respeito da influência que a sua imagem exerce sobre os outros, do julgamento que estes (outros significativos) realizam sobre o indivíduo, somados a uma espécie de auto-sentimento (orgulho ou vergonha) resultante dessa interação social ${ }^{3}$.

Por ser uma estrutura multidimensional o autoconceito é composto por uma série de autorepresentações, tanto central (Self Real) quanto periféricas (Self Real, Self Ideal, Self Desejável, dentre outros), as quais levaram alguns autores a postularem a Teoria da Autodiscrepância ${ }^{4}$. Esta teoria foi proposta a partir da subdivisão de três domínios básicos do self5: a) Self Real (composto pela percepção de traços, características e atributos que o indivíduo possui ou acredita possuir), b) Self Ideal (composto por uma série de aspirações, metas e desejos que o indivíduo idealiza para si) e o Self Desejável (composto por traços, características e atributos desejáveis pela sociedade, tais como responsabilidades, obrigações e condutas que o indivíduo acredita que deveria incorporar à sua autoconstrução).

“Esta teoria postula que os indivíduos são motivados a alcançar uma condição aonde os seus autoconceitos vão ao encontro de suas autorepresentações ${ }^{\prime \prime}$. Assim, a discrepância entre o self real e o self ideal ocorre quando o indivíduo percebe que nunca atingirá determinada meta desejada (ausência de resultados positivos), resultando em estados afetivos de tristeza, desapontamento, desânimo e depressão. Nesse estado, o indivíduo culpa a si mesmo por não conseguir atingir a meta imposta. Já a discrepância entre o self real e o self desejável ocorre quando há a presença (real ou imaginária) de um resultado 
negativo, levando à percepção de que algo terrível irá ocorrer, resultando em medo, ansiedade, tensão e apreensão. Isto ocorre porque os atributos pessoais não se coadunam com o que ele (ou um outro significativo) acredita e considera ser uma obrigação ou dever possuir. Esta percepção de punição gera sentimentos de raiva em relação aos outros e à sociedade ${ }^{6}$.

Dessa forma, um dos primeiros objetivos deste estudo foi utilizar a teoria da autodiscrepância para verificar se a percepção da aparência física de indivíduos obesos insatisfeitos com o peso se modifica, quando estes comparam as percepções atuais de seus corpos (self real) com as projeções que fazem do corpo ideal.

Entretanto, para que o objetivo do estudo fique mais claro, torna-se necessário apresentar uma subdimensão do autoconceito, denominada de autoconceito físico. O autoconceito físico é uma das dimensões do autoconceito global mais fortemente relacionada a este e se subdivide em três aspectos: aparência física, performance motora e controle do peso ${ }^{7}$. A aptidão física e a imagem corporal também são aspectos que compõem este constructo, cabendo à última o aspecto avaliativo da aparência física ${ }^{8,9}$.

Vinculada à aparência física a imagem corporal é a construção cognitiva e afetiva que o indivíduo realiza a respeito de seu próprio corpo ${ }^{10}$. Esta imagem encontra-se estritamente relacionada ao ideal corporal que circula pela mídia na sociedade. Assim, as pessoas aprendem a avaliar seus corpos a partir da interação com o ambiente, sendo, portanto, a auto-imagem desenvolvida e reavaliada continuamente ${ }^{10,11}$.

Na modernidade, o ideal da cultura brasileira são corpos magros, firmes, esbeltos e delineados. Aqueles cujos corpos não se encontram dentro desta condição e não atingem este ideal cultural, podem desenvolver uma imagem corporal negativa de si, resultando em baixa auto-estima e depressão ${ }^{12}$. Apesar da pressão desenvolvida pela cultura na busca do corpo ideal magro, firme e delineado, a obesidade é universal, de prevalência crescente e vem adquirindo proporções alarmantes, inclusive em países que, paradoxalmente, ainda sofrem os efeitos da fome e da desnutrição crônica ${ }^{13-17}$.

Observa-se, assim, que há uma contradição entre o aumento da obesidade e o padrão cultural de beleza prescrito pela cultura brasileira. Como o padrão de beleza acaba por interferir nas projeções individuais do corpo ideal, o foco do presente estudo foi avaliar a discrepância entre a imagem real e a imagem ideal de indivíduos obesos insatisfeitos com o corpo, considerando que a discrepância entre essas imagens pode levá-los a estados afetivos negativos de desânimo, tristeza e depressão. Além disso, este estudo procurou detectar as possíveis alterações na dieta que os indivíduos obesos fariam, a fim de atingir o corpo ideal.

\section{MÉ TO D O S}

Por se tratar de estudo exploratório, utilizando uma teoria da psicologia social para explicar um contexto de obesidade, a amostra foi de conveniência, composta por 25 sujeitos obesos de ambos os sexos, cujo único critério de exclusão foi possuir índice de massa corporal (IMC) ${ }^{18,19}$ inferior a $30,0 \mathrm{~kg} / \mathrm{m}^{2}$. Todos os sujeitos concordaram em participar, assinando o termo de consentimento livre e esclarecido, no qual foi explicado o propósito do estudo, bem como, assegurado o sigilo da identidade.

Os instrumentos utilizados foram a Escala de Aparência Física (EAF) e o Questionário de Prioridade Alimentar (QPA). A Escala de Aparência Física (EAF) é composta por 10 itens que avaliam partes corporais, utilizando escores que variam de 1 (parte corporal de pequena proporção) a 5 (parte corporal de grande proporção). Como algumas partes corporais diferem para homens (tórax) e mulheres (seios), esta escala foi elaborada em duas versões - a que se aplica aos homens e a que se aplica às mulheres.

Como não foram encontradas na literatura escalas que permitissem avaliar a aparência física real e ideal, esta escala foi idealizada para o 
presente estudo (Tabela 1). Os processos de validação e precisão da EAF encontram-se na seção resultados.

O Questionário de Prioridade Alimentar (QPA) é um instrumento elaborado com o objetivo de avaliar a importância atribuída aos principais alimentos que compõem a dieta dos brasileiros. Este questionário apresenta uma série de alimentos (92 itens) dispostos em grupos como Pães, Doces, Bebidas etc. Ao respondente é solicitado que, utilizando uma escala de cinco pontos ( $1=$ nada importante a $5=$ muito importante), indique o grau de importância que atribui a cada alimento em sua dieta. Este questionário foi elaborado, somente, para agrupar, posteriormente, os alimentos de acordo com as diferentes categorias previstas pela pirâmide alimentar ${ }^{20}$.

A EAF e o QPA foram elaborados em duas versões que diferiam quanto às suas instruções. Em uma primeira versão, as instruções solicitavam ao respondente que utilizasse a EAF e o QPA para avaliar, respectivamente, a sua aparência física atual e descrevesse a importância atribuída aos alimentos na sua dieta atual. Na segunda versão, as instruções solicitavam ao respondente que utilizasse a escala (EAF) e o questionário (QPA) para descreverem, respectivamente, as suas projeções de um corpo ideal e a importância atribuída aos alimentos para a aquisição deste corpo ideal.

Os dados foram coletados individualmente e ao longo de um mês por uma das pesquisadoras,

Tabela 1. Itens e cargas fatoriais obtidos na validação da escala de aparência física. Brasília (DF), 2006.

\begin{tabular}{llc}
\hline Número & Itens & Carga Fatorial \\
\hline 1 & Coxas & 0,65 \\
2 & Largura dos quadrícepes & 0,57 \\
3 & Definição da panturrilha & 0,56 \\
4 & Definição das coxas & 0,53 \\
5 & Panturrilha & 0,44 \\
6 & Peso & 0,44 \\
7 & Definição do antebraço & 0,42 \\
8 & Volume do abdome & 0,39 \\
9 & Aparência dos seios* & 0,37 \\
10 & Glúteos & 0,36 \\
\hline
\end{tabular}

*O item 9 só deverá ser incorporado à escala de aparência física quando for aplicada às mulheres. a qual apresentava inicialmente a primeira versão dos instrumentos e após a sua finalização, apresentava a segunda versão. Os indivíduos levaram em torno de 15 a 20 minutos para responder os questionários.

Para a validação e a análise da precisão da EAF foi utilizada a Análise Fatorial (Principal Axis Factoring, rotação Oblimin, cargas fatoriais iguais ou superiores a 0,35$)^{21}$ e para avaliar o índice de precisão interna do fator utilizou-se o Alfa de Cronbach ${ }^{22}$. Em todas as análises foi utilizado erro de $5 \%$.

Para avaliar se a percepção do corpo atual diferia da projeção do corpo ideal, foi realizado o teste $t$ pareado, utilizando-se o fator Aparência Física da EAF como variável dependente.

Em relação à dieta, os alimentos que compõem o QPA foram agrupados nas categorias determinadas pela pirâmide alimentar (frutas, hortaliças, carnes, cereais, leite e produtos lácteos, legumes, açúcares e doces, óleos e gorduras). Alguns alimentos foram analisados separadamente (café, refrigerante, isotônicos) por não estarem inseridos em nenhuma das categorias da pirâmide. O escore do indivíduo em cada categoria foi obtido a partir da média aritmética, ou seja, somando-se todos os alimentos que compõem a categoria e dividindo-se pelo número de alimentos que esta contém. Uma vez obtidas as médias dos indivíduos para cada categoria da pirâmide, foi realizado o teste $t$ pareado para avaliar se haviam e quais seriam as mudanças dietéticas determinadas pelos indivíduos, com a finalidade de atingir o corpo ideal.

Este trabalho está de acordo com a Resolução 196/196 do Conselho Nacional de Saúde, que regulamenta as pesquisas envolvendo seres humanos, tendo obtido aprovação pelo Comitê de Ética em Pesquisa da Universidade Católica de Brasília com seres humanos.

\section{RESULTADOS}

Para melhor compreensão, os resultados serão apresentados em duas seções: a) Validação 
e precisão da EAF e b) Análise das percepções das imagens e dietas real e ideal.

a) Validação e precisão da EAF:

A escala foi aplicada a uma amostra de 220 indivíduos, sendo 92,00\% do sexo feminino, com idade média de 32 anos (desvio-padrão $D P=5,67)$. Os testes iniciais para avaliar a estrutura fatorial do instrumento demonstraram os seguintes resultados: a) Kaiser-Meyer-Olkin $(\mathrm{KMO})=0,68$; b) Bartlett's Test of Sphericity (231) $=918,86$; $p=0,001$; c) eigenvalue $>1,5=3$. Optou-se pela extração de um único fator, denominado de Aparência Física, cuja variância total foi de 16,33\% e índice de precisão interna (Alfa de Cronbach) igual a 0,74 para as mulheres e 0,73 para os homens. Estes resultados revelam que a Escala de Aparência Física (EAF) encontra-se dentro dos padrões psicométricos, podendo ser utilizada em estudos científicos.

b) Análise das percepções das imagens e dietas real e ideal:

Para avaliar diferenças entre as imagens real e ideal de indivíduos obesos, assim como entre as dietas real e ideal, participaram da amostra 25 sujeitos, com peso médio igual a $98,24 \mathrm{~kg}$ ( $D P=4,23)$, estatura média igual a $167(D P=4,61)$, IMC médio igual a 35,42 (DP=4,86), sendo 76\% do sexo feminino com $2^{\circ}$ grau completo $(48 \%)$ e idade média de 39,24 ( $\mathrm{DP}=5,01)$.

O resultado demonstrou que as percepções diferem entre si $[t(24)=2,16 ; p=0,04]$, apresentando o corpo ideal $(3,22 \mathrm{DP}=0,24)$ melhor aparência física do que o corpo real $(2,99$ $\mathrm{DP}=0,40)$.

Com relação à dieta, os resultados demonstraram que os sujeitos entrevistados atribuiram maior importância às frutas $[t(24)=-5,42 ; p=0,001]$ e às hortaliças [ $t(24)=-2,95 ; p=0,007]$. De acordo com os testes eles diminuiriam a ingestão de carnes $[t(24)=3,83 ; p=0,001]$ e café $[t(24)=3,76$; $p=0,001]$ e não modificariam o hábito alimentar em relação aos cereais $[t(24)=-0,50 ; p=0,62]$, ao leite e aos produtos lácteos $[t(24)=0,48 ; p=0,64]$, aos legumes $[t(24)=-1,24 ; p=0,23]$, aos açúcares
Tabela 2. Médias (M), desvios-padrão (DP) e níveis de significância $(p)$ obtidos para as categorias da pirâmide alimentar de indivíduos obesos. Brasília (DF), 2006.

\begin{tabular}{|c|c|c|c|c|c|}
\hline \multirow{2}{*}{ Categorias } & \multicolumn{2}{|c|}{ Dieta real } & \multicolumn{2}{|c|}{ Dieta ideal } & \multirow{2}{*}{$p$} \\
\hline & $\mathrm{M}$ & DP & $\mathrm{M}$ & $\mathrm{DP}$ & \\
\hline Cereais & 2,39 & 0,37 & 2,43 & 0,37 & $p=0,620$ \\
\hline Frutas & 2,41 & 0,50 & 3,03 & 0,54 & $p=0,001$ * \\
\hline Legumes & 2,23 & 0,59 & 2,37 & 0,64 & $p=0,230$ \\
\hline Doces & 2,32 & 0,65 & 2,16 & 0,47 & $p=0,240$ \\
\hline Laticínios & 2,42 & 0,48 & 2,36 & 0,39 & $p=0,640$ \\
\hline Carnes & 2,63 & 0,41 & 2,11 & 0,43 & $p=0,001$ * \\
\hline Hortaliças & 2,41 & 0,75 & 3,03 & 0,75 & $p=0,007^{*}$ \\
\hline Óleos & 2,27 & 0,64 & 1,96 & 0,60 & $p=0,110$ \\
\hline Café & 2,28 & 1,28 & 1,36 & 0,57 & $p=0,001$ * \\
\hline Refrigerante & 2,72 & 1,24 & 2,12 & 1,27 & $p=0,120$ \\
\hline Isotônico & 2,08 & 1,08 & 2,36 & 1,29 & $p=0,410$ \\
\hline
\end{tabular}

${ }^{*} p<0,05$.

e aos doces [ $t(24)=1,20 ; p=0,24]$, aos óleos e às gorduras $[t(24)=1,64 ; p=0,11]$, aos refrigerantes $[t(24)=1,62 ; p=0,12]$ e aos isotônicos $[t(24)=-0,84$; $p=0,41]$. A Tabela 2 apresenta as médias, os desvios-padrão e os níveis de significância $(p)$ encontrados para a dieta real e a dieta ideal.

\section{DISCUSSÃ O}

Os indivíduos obesos avaliados apresentam uma discrepância na imagem, projetando um corpo ideal, cuja aparência física é mais positiva do que a aparência atual. Ainda que não avaliado neste estudo, pode-se esperar que esta discrepância entre imagem atual e imagem ideal desencadeará distúrbios afetivos típicos, tais como, tristeza, desânimo e depressão.

Na cultura ocidental, ser magra, principalmente para a mulher, simboliza competência, sucesso, controle e atrativos sexuais, enquanto a aparência obesa e o excesso de peso representam preguiça, indulgência pessoal, falta de autocontrole e de força de vontade. Sendo assim, a obesidade oferece uma conotação pejorativa aos indivíduos, em especial às mulheres, sendo uma explicação possível para a insatisfação pessoal'.

A mídia também contribui para esta realidade, pois induz o indivíduo a buscar o corpo ideal 
e a beleza física. Assim, a cultura e os avanços tecnológicos são agentes responsáveis pela promoção do padrão de beleza magro, reforçando crenças culturais sobre a importância do controle de peso. Os hábitos alimentares, por outro lado, são construídos com base em determinações socioculturais, nas quais a mídia desempenha papel estruturador na construção e desconstrução de procedimentos alimentares ${ }^{23}$.

Embora os veículos de comunicação reforcem o padrão de beleza magro, há um paradoxo entre o que é veiculado e o padrão comportamental e alimentar da maioria dos indivíduos que sofrem a ação da mídia. Como exemplo, pode-se citar um estudo com adolescentes ${ }^{24}$, no qual foi verificada a presença de associação direta entre o tempo de assistir televisão e o aumento da ingestão de alimentos energéticos, refrigerantes e baixa ingestão de frutas e hortaliças, proporcionando, assim, o aumento descontrolado da obesidade.

O ser humano tem necessidade fisiológica de se alimentar, mas seu funcionamento psíquico e o contexto sociocultural modulam o comportamento. Seu funcionamento psíquico determinará a relação com o alimento, suas preferências e como organizará a rotina de suas refeições. Por outro lado, o contexto sociocultural contribuirá com costumes, tipos de alimentos, tradições no preparo, além de mediar a sua oferta e a disponibilidade $^{25}$.

Diante dessa influência, a sociedade e a cultura incentivam o consumo de alimentos não saudáveis, veiculando-os como práticos e rápidos, artifício este que atinge a sociedade atual, que carece de tempo para o preparo dos alimentos ${ }^{26}$. $O$ incentivo ao consumo desses alimentos vem acarretando o aumento da obesidade, cujas conseqüências são prejudiciais para o corpo físico e para o estado psicológico dos indivíduos.

No intuito de incorporar mudanças nos hábitos alimentares, os nutricionistas utilizam estratégias de comportamento alimentar e fazem uso de instrumentos que valorizam a prática ali- mentar saudável. Dentre estes instrumentos (inquéritos, registros alimentares, análise sensorial), a pirâmide alimentar ${ }^{20}$ é o guia de alimentação saudável mais utilizado para este fim, a qual partindo da quantidade e qualidade dos alimentos verifica se os hábitos alimentares podem ser considerados como saudáveis ou não.

Com base nas categorias estipuladas pela pirâmide alimentar, os resultados demonstraram que os indivíduos obesos não mudariam os seus hábitos em relação aos cereais, às leguminosas, aos açúcares e aos doces, ao leite e aos produtos lácteos, aos óleos e às gorduras, aos refrigerantes e aos isotônicos. Dentre estes alimentos, os açúcares e os doces, os óleos e as gorduras são alimentos que se encontram no topo da pirâmide, a qual prevê consumo parcimonioso. Esses alimentos são facilitadores de aumento ponderal e acúmulo expressivo de tecido adiposo.

Os resultados também demonstram aumento no consumo de frutas e hortaliças, bem como, diminuição da ingestão de carnes e café preto. Estes resultados vão ao encontro de uma mudança nos hábitos alimentares, uma vez que o aumento da ingestão de frutas e hortaliças contribui para o funcionamento e a metabolização orgânica, peristalse regular do intestino, prevenindo neoplasias como, por exemplo, câncer de cólon. Cada fruta, por sua vez, tem a sua vitamina principal, sendo, portanto, indispensável variá-las para garantir a ingestão suficiente das diferentes vitaminas. Assim, recomenda-se o consumo diário de 3 a 4 porções variadas de frutas após as principais refeições ou nos intervalos ${ }^{27}$.

A diminuição acentuada da ingestão de carnes pode afetar o crescimento, a regeneração e a renovação de tecidos (ossos, músculos, pele, cabelos, unhas), bem como a produção de hormônios, enzimas e a integridade do sistema imunológico. Entretanto, o consumo excessivo sugere a produção de quantidades indesejáveis de uréia e de outros compostos que podem representar uma sobrecarga nas funções hepática e renal. Por essa razão, o catabolismo protéico excessivo eleva as necessidades hídricas do organismo ${ }^{28}$, necessi- 
tando, portanto, de um balanço equilibrado em relação ao seu consumo.

A referência à diminuição do café preto nos resultados, indiretamente oferece benefício ao funcionamento orgânico, pois esta é uma bebida que, em sua composição, possui cafeína, substância cujos efeitos estão associados ao aumento da excreção de cálcio, contribuindo para a osteoporose $\mathrm{e}^{29}$.

Os resultados, portanto, demonstraram que o aumento do consumo de frutas e hortaliças, sem que haja a diminuição do consumo de alimentos inseridos nas categorias do topo da pirâmide (açúcares e doces, óleos e gorduras) não levaria à diminuição do peso e, tampouco, a atingir o corpo ideal. Também, observa-se que não há a valorização do comportamento alimentar saudável, pois os indivíduos permaneceriam com os mesmos hábitos, ou seja, ingerindo alimentos inseridos em categorias do topo da pirâmide, acreditando, com isso, obter o corpo ideal, sem se preocupar com a saúde.

A prevalência considerável da obesidade é conseqüência do comportamento alimentar totalmente inadequado, o qual está relacionado ao sedentarismo, ao elevado consumo de alimentos altamente energéticos, tais como fast food, à substituição das refeições por sanduíches, ao elevado consumo de bebidas carbonatadas e, principalmente, à ausência do apoio familiar para o consumo de uma dieta saudável$\left.\right|^{30}$.

A seleção de alimentos tem sido relacionada à obesidade, não somente em função do volume da ingestão alimentar, mas também em relação à composição e à qualidade da dieta, pois os padrões alimentares vêm se modificando, devido ao aumento no consumo de guloseimas (bolachas recheadas, salgadinhos, doces) e refrigerantes ${ }^{31}$.

Assim, o desconhecimento do comportamento alimentar saudável, o descontrole da saciedade e o desejo de comer, levam à obesidade e, conseqüentemente, a uma visão negativa do corpo, desencadeando discrepâncias da percepção corporal e resultando em alterações psicossomáticas $^{32}$. De acordo com a teoria da autodiscrepância pode-se esperar que quanto maior for a discrepância, maior será o distúrbio afetivo apresentado pelo indivíduo, neste caso, resultando em tristeza, desânimo e depressão.

Além disso, os resultados permitem afirmar que o desconhecimento dietético dos participantes do estudo desencadeia um efeito em círculo, no qual o indivíduo não emagrece por não mudar os seus hábitos alimentares e com isso não se aproxima do corpo idealizado o que, por sua vez, gera um processo depressivo que induz ao aumento da ingestão energética, acentuando a obesidade. Sem a intervenção de um nutricionista, que venha orientar e introduzir, paulatinamente, mudanças nos hábitos alimentares, este processo não pode ser interrompido.

Cabe, ainda, ao nutricionista não apenas orientar a dieta com vistas ao alcance do corpo ideal, mas também, orientar a dieta visando à saúde. Apesar de não ter sido foco deste estudo, a saúde do corpo deve ser priorizada muito antes da busca de um corpo ideal, fruto do estereótipo sócio-cultural. Resta, entretanto, que novos estudos sejam realizados buscando avaliar os demais pressupostos da teoria da autodiscrepância no contexto da nutrição, tais como, verificar o estado afetivo de obesos que apresentam alta discrepância entre as imagens atual e ideal, ou avaliando se as percepções da aparência física e da dieta são diferentes entre obesos insatisfeitos e satisfeitos com o seu peso atual.

\section{COLABORADORES}

P. KANNO realizou a revisão bibliográfica, elaborou os instrumentos de pesquisa e coletou os dados. M. RABELO participou da coleta e da digitação dos dados. G.F. MELO realizou as análises estatísticas do estudo. A. GIAVONI orientou o estudo, a psicometria e as análises estatísticas.

\section{REFERÊ NCIAS}

1. Costa PCG. Escala de autoconceito no trabalho: construção e validação. Rev Psicol: Teoria e Pesquisa. 2002; 18(1):75-81. 
2. Villa AS, Auzmendi E. Medição do autoconceito. São Paulo: INEP; 1999.

3. Giavoni A, Tamayo A. Inventário dos esquemas de gênero do autoconceito (IEGA). Rev Psicol: Teoria e Pesquisa. 2000; 16(2):175-84.

4. Bybee JA, Zigler E. Self-image and guilt: a further test of the cognitive developmental formulation. J Pers. 1991; 59(4):733-45.

5. Strauman TJ, Higgins ET. Self-discrepancies as predictors of vulnerability to distinct syndromes of chronic emotional distress. J Pers. 1988; 56(4): 685-707.

6. Higgins ET, Bond K, Strauman TJ. Self discrepancies and emotional vulnerability: how magnitude, accessibility, and type of discrepancy influence affect. J Per Soc Psy. 1986; 51(1):5-15.

7. Franzoi SL, Shields SA. The body esteem scale: multidimensional structure and sex differences in a college population. J Per Ass. 1984; 48(2):173-8.

8. Nogueira MR. Inventário de autoconceito físico (IAF) [dissertação]. Brasília: Universidade Católica de Brasília; 2004.

9. Marsh HW, Richards GE, Johnson S, Tremayne P. Physical self description questionnaire: psychometric properties and a multitrait: multimethod analysis of relations to existing instruments. J Sport Exerc Psychol. 1994; 16(3):270-305.

10. Tavares MCC. Imagem corporal: conceito e desenvolvimento. São Paulo: Manole; 2003.

11. Becker JRB. Manual de psicologia aplicada ao exercício \& esporte. Porto Alegre: Edelbra; 1999.

12. Cash, TF. Body images attitudes among obese enrollees in a commercial weight loss program. Percep Mot Skills. 1993; 77(3):1099-103.

13. Monteiro CA, Benício MH, lunes R, Gouveia NC. Nutritional status of Brazilian children: trends from 1975 to 1989. Bull World Health Organ. 1992; 70(5):657-66.

14. Felippe F, Santos AM. Novas demandas profissionais: obesidade em foco. Rev ADPPUCRS. 2004; (5):63-70.

15. Bernardi F, Cichelero C, Vitolo MR. Comportamento de restrição alimentar e obesidade. Rev Nutr. 2005; 18(1):85-93.

16. Campos LA, Leite AJM, Almeida PC. Nível socioeconômico e sua influência sobre a prevalência de sobrepeso e obesidade em escolares adolescentes do município de Fortaleza. Rev Nutr. 2006; 19(5): 531-8.

17. Pinheiro ARO, Freitas SFT, Corso ACT. Uma abordagem epidemiológica da obesidade. Rev Nutr. 2004; 17(4):523-33.
18. World Health Organization. Obesity: preventing and managing the global epidemic. Report of a WHO Consultation on Obesity. Geneva: World Health Organization; 1998.

19. Velásquez-Meléndez G, Pimenta AM, Kac G. Epidemiologia do sobrepeso e da obesidade e seus fatores determinantes em Belo Horizonte (MG), Brasil: estudo transversal de base populacional. Rev Panam Salud Publica. 2004; 16(5): 308-14.

20. Philippi ST, Latterza AR, Cruz ATR, Ribeiro LC. Pirâmide alimentar adaptada: guia para escolha dos alimentos. Rev Nutr. 1999; 12(1):65-80.

21. Harman HH. Modern factor analysis. Chicago: University of Chicago Press; 1976.

22. Cronbach LJ. Fundamentos da testagem psicológica. Porto Alegre: Artes Médicas; 1996.

23. Serra GM, Santos AEM. Saúde e mídia na construção da obesidade e do corpo perfeito. Ciênc Saúde Colet. 2003; 8(3):691-701.

24. Coon KA, Tucker KL. Television and children's consumption patterns. A review of the literature. Minerva Pediatr. 2002; 54(5):423-36.

25. Perez GH, Romano BW. Comportamento alimentar e síndrome metabólica: aspectos psicológicos. Rev Soc Cardiol. 2004; 14(4):544-50.

26. Santos AM. Novas demandas profissionais: obesidade em foco. Rev ADPPUCRS. 2004; 5:63-70.

27. Sigulem DM, Devincenzi MU, Lessa AC. Diagnóstico do estado nutricional da criança e do adolescente. J Ped. 2000; 76(3):275-84.

28. McArdle WD, Katch Fl, Katch VI. Fisiologia do exercício: energia, nutrição e desempenho humano. Rio de Janeiro: Guanabara Koogan; 1998.

29. Gali JC. Osteoporose. Acta Otop Bras. 2001; 9(2): 53-62.

30. Vieira MA, Atihe RD, Soares RC, Damiani D, Setia N. Adesão inicial ao tratamento de adolescentes obesos em programas de redução de peso. Pediatria. 2004; 26(4):240-6.

31. Triches RM, Giugliani ERJ. Obesidade, práticas alimentares e conhecimentos de nutrição em escolares. Rev Saúde Pública. 2005; 39(4):541-7.

32. Vasques F, Martins FC, Azevedo AP. Aspectos psiquiátricos do tratamento da obesidade. Rev Psiq Clin. 2004; 31(4):195-8.

Recebido em: 24/10/2006

Versão final reapresentada em: 9/8/2007 Aprovado em: 29/2/2008 